\title{
Pan-IDH Mutant Inhibitor AG-881
}

National Cancer Institute

\section{Source}

National Cancer Institute. Pan-IDH Mutant Inhibitor AG-881. NCI Thesaurus. Code C122874.

An orally available inhibitor of mutated forms of both isocitrate dehydrogenase type 1 (IDH1, IDH1 [NADP+] soluble) in the cytoplasm and type 2 (IDH2, isocitrate dehydrogenase $[\mathrm{NADP}+]$, mitochondrial) in the mitochondria, with potential antineoplastic activity. Upon administration, pan-IDH mutant inhibitor AG-881 specifically inhibits mutant forms of IDH1 and IDH2, thereby inhibiting the formation of the oncometabolite 2-hydroxyg lutarate (2HG) from alpha-ketoglutarate (a-KG). This prevents 2HG-mediated signaling and leads to both an induction of cellular differentiation and an inhibition of cellular proliferation in tumor cells expressing IDH mutations. In addition, AG-881 is able to penetrate the blood-brain barrier (BBB). IDH1 and 2, metabolic enzymes that catalyze the conversion of isocitrate into a-KG, play key roles in energy production and are mutated in a variety of cancer cell types. In addition, mutant forms of IDH 1 and 2 catalyze the formation of $2 \mathrm{HG}$ and drive cancer growth by blocking cellular differentiation and inducing cellular proliferation. 